TRANSACTIONS OF THE

AMERICAN MATHEMATICAL SOCIETY

Volume 315, Number 2, October 1989

\title{
PIXLEY-ROY HYPERSPACES OF $\omega$-GRAPHS
}

\author{
J. D. MASHBURN
}

\begin{abstract}
ABSTRACr. The techniques developed by Wage and Norden are used to show that the Pixley-Roy hyperspaces of any two $\omega$-graphs are homeomorphic. The Pixley-Roy hyperspaces of several subsets of $\mathbf{R}^{n}$ are also shown to be homeomorphic.
\end{abstract}

\section{INTRODUCTION}

Since it was introduced in 1969 , the Pixley-Roy hyperspace, $\operatorname{PR}[X]$, of a topological space $X$ has been intensely studied with the hope of establishing how the properties of $X$ affect those of $\mathrm{PR}[X]$. This study has met with some success, especially in the area of cardinal functions. However, there is a class of questions which, until recently, eluded investigators: For which spaces $X$ and $Y$ will $\operatorname{PR}[X]$ be homeomorphic to $\operatorname{PR}[Y]$ ? For several years the only results in this area were some embedding results obtained by van Douwen [vD] and Lutzer [L]. In 1985 Wage [W] achieved a breakthrough by developing a technique for breaking up neighborhoods around points in certain spaces which allowed him to define homeomorphisms between those neighborhoods. Using this technique he was able to show that Pixley-Roy hyperspaces of spaces like $R$ or $[0,1]$ are homogeneous. In 1986 Norden [N] extended Wage's technique to one which broke up an entire space. With this he was able to show that the Pixley-Roy hyperspaces of any two $P$-graphs (one-dimensional polyhedra with a finite number of points removed) are homeomorphic. It follows that the PixleyRoy hyperspaces of spaces like $R,[0,1]$, and the circle are all homeomorphic. It is the purpose of this paper to use Norden's technique to show that Pixley-Roy hyperspaces of infinite, as well as finite, graphs are all the same.

Definition. A $T_{2}$ space $X$ with no isolated points is an $\omega$-graph if there is a countable discrete subset $D$ of $X$ and a countable collection $I$ of pairwise disjoint copies of $(0,1)$ such that $X \backslash D=\bigcup \mathbf{I}, \mathbf{I}$ is locally finite on $X$, and for every $x \in D,\{x\} \cup(\bigcup\{I \in \mathbf{I}: x \in \bar{I}\})$ is a neighborhood of $x$ which can be embedded in $\mathbf{R}^{2}$. The set $D$ is called a dividing set for $X$.

The main result of this paper can be stated as follows.

Received by the editors March 10, 1988.

1980 Mathematics Subject Classification (1985 Revision). Primary 54B20; Secondary 54F65.

Supported by a Summer Research Grant from the University of Dayton. 
Theorem 1. If $X$ and $Y$ are $\omega$-graphs then $\operatorname{PR}[X]$ is homeomorphic to $\operatorname{PR}[Y]$.

$\S$ II will consist of preliminary definitions, notation, and observations necessary for the proof of the Theorem 1 . Theorem 1 will be proved in $\S I I I$, and $\S I V$ will contain some related results.

We will use $\operatorname{PR}[X]$ to denote the Pixley-Roy hyperspace of $X$. Our notation for the open subsets of $\mathrm{PR}[X]$ will be standard. We will use $F[A]$ to denote the set of nonempty finite subsets of a set $A$, and $F^{\prime}[A]$ to denote the set of all finite subsets of $A$. The notation " $X \approx Y$ " will mean that $X$ is homeomorphic to $Y$.

\section{Preliminary matters}

Let $X$ be an $\omega$-graph and let $X_{0}$ be a dividing set for $X$. Enumerate $X_{0}$ as $\left\{x_{n}: n<\omega\right\}$. Let $\mathbf{I}_{0}$ be the countable collection of pairwise disjoint copies of $(0,1)$ whose union makes up $X \backslash X_{0}$. We may assume that every element of $I_{0}$ has at least one endpoint in $X_{0}$. For each $n<\omega$ let $\mu(n)$ be the number of elements of $X \backslash X_{0}$ having $x_{n}$ as an endpoint. For each $I \in \mathbf{I}_{0}$, fix a linear structure and orientation for $I$. Let $Q_{0}$ be the set of all midpoints of elements of $\mathbf{I}_{0}$ and, for each $p \in X_{0}$, let $O_{p}$ be the component of $X \backslash Q_{0}$ containing $p$. Then $Q_{0}$ is a discrete subset of $X$ and $O_{p} \cap O_{q}=\varnothing$ if $p \neq q$.

For each $p \in X_{0}$ and each $I \in \mathbf{I}_{0}$ having $p$ as an endpoint, choose a sequence of points in $I \cap O_{p}$ converging monotonically to $p$. This can be done because each element of $X_{0}$ is the endpoint of at least one element of $\mathbf{I}_{0}$. Let $Q_{1}$ be the set of all points of $X$ which are elements either of $Q_{0}$ or of the sequences just chosen. Call $Q_{1}$ the 1 st cut-set of $X$. Set $\widehat{Q}_{1}=Q_{1}$. Let $\mathbf{I}_{1}$ be the countable collection of pairwise disjoint copies of $(0,1)$ whose union makes up $X \backslash\left(\widehat{Q}_{1} \cup X_{0}\right)$. Call $I_{1}$ the set of intervals in $X$ derived from $\widehat{Q}_{1}$.

Assume that $n<\omega$, that $Q_{n}$ is a discrete subset of $X \backslash X_{0}$, and that $\mathbf{I}_{n}$ is a countable collection of pairwise disjoint intervals in $X$. Let $Q_{n+1}$, the $(n+1)$ th cut-set of $X$, be the set of midpoints of elements of $\mathbf{I}_{n}$ and let $\widehat{Q}_{n+1}=\widehat{Q}_{n} \cup Q_{n+1}$. Let $\mathbf{I}_{n+1}$, the set of intervals in $X$ derived from $\widehat{Q}_{n+1}$, be the countable collection of pairwise disjoint copies of $(0,1)$ whose union makes up $X \backslash\left(\widehat{Q}_{n+1} \cup X_{0}\right)$. Set $Q=\bigcup_{n<\omega} Q_{n}$.

For every $1 \leq m<\omega$ and every $n<\omega$, let $\mathbf{I}_{m, n}=\left\{I \in \mathbf{I}_{m}: I \subset O_{x_{n}}\right\}$. This is the set of those elements of $\mathbf{I}_{m}$ which "cluster" around $x_{n}$.

For every $1 \leq n<\omega$ let $\Sigma(n)$ be the set of sequences, $\sigma$, defined on $n+1$ such that $\sigma(0), \sigma(1) \in \omega$ and $\sigma(m) \in\{0,1\}$ for all $1<m \leq n$. Let $m<\omega$. Since $I_{1, m}$ is countable, it can be enumerated as $\left\{I_{\langle m, n\rangle}: n<\omega\right\}$. In this way the set $\mathbf{I}_{1}$ is indexed by $\Sigma(1)$. Assume that the elements of $\Sigma(n)$ have been used to index the elements of $\mathbf{I}_{n}$. Let $I \in \mathbf{I}_{n+1}$. There is a unique $\sigma \in \Sigma(n)$ such that $I \subset I_{\sigma}$. If $I$ is the left-hand half of $I_{\sigma}$, then let $\tau$ be the element of $\Sigma(n+1)$ such that $\tau \mid n+1=\sigma$ and $\tau(n+1)=0$ and set $I_{\tau}=I$. If $I$ is the right-hand half of $I_{\sigma}$, then let $\tau$ be the element of $\Sigma(n+1)$ such that $\tau \mid n+1=\sigma$ and $\tau(n+1)=1$ and set $I_{\tau}=I$. Let $\Sigma=\bigcup_{1 \leq n<\omega} \Sigma(n)$. 
The following lemma consists of observations which are immediate consequences of the previous definitions and its proof is omitted.

Lemma 2. Let $1 \leq m \leq n<\omega$.

1. If $I \in \mathbf{I}_{n}$ then $I \cap Q_{m} \neq \varnothing$.

2. If $p \in Q_{m}$ then there are exactly two elements, $I_{1}$ and $I_{2}$, of $\mathbf{I}_{n}$ such that $p$ is an endpoint of both $I_{1}$ and $I_{2}$. Furthermore, $I_{1} \cup I_{2} \cup\{p\}$ is open in $X$.

3. If $I \in \mathbf{I}_{m}$ then there are exactly two elements of $\mathbf{I}_{m+1}$ that are subintervals of $I$.

4. If $I_{\sigma} \in \mathbf{I}_{n}$ then there is exactly one element, $I_{\sigma \mid m+1}$, of $\mathbf{I}_{m}$ that contains $I_{\sigma}$.

5. If $\sigma \in \Sigma(1), \sigma(0)=k$, and $\sigma(1)=1$, then $I_{\sigma}$ is the lth element of $\mathbf{I}_{1, k}$.

6. If $J_{\sigma} \in \mathbf{I}_{n, k}$ then $\sigma \in \Sigma(n)$ and $\sigma(0)=k$.

7. For any $n, k<\omega,\left\{\operatorname{Int}\left[\mathrm{Cl}\left(\bigcup\left\{\mathbf{I}_{\sigma} \in I_{n, k}: \sigma(1)>a\right\}\right)\right]: a<\omega\right\}$ forms $a$ local base for $x_{k}$.

For each $p \in X$ and each $1 \leq n<\omega$ let $\mathbf{A}_{n}(p)=\left\{I \in \mathbf{I}_{n}: p \in \bar{I}\right\}$ and let $\mathbf{A}_{n}^{*}(p)=\bigcup \mathbf{A}_{n}(p)$. If $p \in Q_{n}$ then $\mathbf{A}(p)$ and $\mathbf{A}^{*}(p)$ will denote $\mathbf{A}_{n+1}(p)$ and $\mathbf{A}_{n+1}^{*}(p)$ respectively. If $B \in \operatorname{PR}[X]$ then set $\mathbf{A}_{n}(B)=\bigcup_{p \in B} \mathbf{A}_{n}(p)$ and $\mathbf{A}_{n}^{*}(p)=\bigcup_{p \in B} \mathbf{A}_{n}^{*}(p)$. If $B \in F\left[Q_{n}\right]$ then set $\mathbf{A}(B)=\bigcup_{p \in B} \mathbf{A}(p)$ and $\mathbf{A}^{*}(B)=$ $\bigcup_{p \in B} \mathbf{A}^{*}(p)$.

Set $M_{0}=\{\varnothing\}$ and, for each $1 \leq n<\omega$, let $M_{n}=\left\{E \in F\left(\widehat{Q}_{n}\right): E \cap Q_{m} \neq \varnothing\right.$ for all $1 \leq m \leq n\}$. For $1 \leq n<\omega$ call $M_{n}$ the set of elements of $\operatorname{PR}[X]$ compatible with $\hat{Q}_{n}$. Note that if $m>n$ and $E \in M_{n}$ then $E \cap Q_{m}=\varnothing$. Also, if $k \neq l$ then $M_{k} \cap M_{l}=\varnothing$. For each $n<\omega$ and each $E \in M_{n}$, let $S_{E}=\{A \in$ $\left.\mathrm{PR}[X]: A \cap \widehat{Q}_{n+1}=E\right\}$. Thus, if $A \in S_{E}$ and $E \in M_{n}$, then $A \cap Q_{n+1}=\varnothing$. The set $\left\{S_{E}: E \in M\right\}$ where $M=\bigcup_{n<\omega} M_{n}$ is a partition of $\operatorname{PR}[X]$ and is called the fundamental partition of PR[X] based on $M$. If $E \in M_{n}$ then $S_{E}$ can be written as $\left\{A \cup B \cup E: A \in F^{\prime}\left[X_{0}\right]\right.$ and $\left.B \in F^{\prime}\left[X \backslash\left(\widehat{Q}_{n+1} \cup X_{0}\right)\right]\right\}$. Recall that $X \backslash\left(\widehat{Q}_{n+1} \cup X_{0}\right)=\bigcup \mathbf{I}_{n+1}$.

For each $E \in M_{n}$ let $\widehat{F}_{E}=\left\{I \in \mathbf{I}_{n+1}: I \subset A^{*}(E)\right\}$. If $n \geq 2$, let $E^{\prime}=$ $E \backslash Q_{n}=E \cap \widehat{Q}_{n-1}$. If $n \geq 3$ then $E^{\prime \prime}$ is $E \cap \widehat{Q}_{n-2}$. If $n=2$ then set $E^{\prime \prime}=\varnothing$.

Now let $Y$ be another $\omega$-graph and let $Y_{0}$ be a dividing set for $Y$. Enumerate $Y_{0}$ as $\left\{y_{n}: n<\omega\right\}$. Then the function $\lambda: X_{0} \rightarrow Y_{0}$ given by $\lambda\left(x_{n}\right)=y_{n}$ is a bijection. Let $\mathbf{J}_{0}$ be a countable collection of pairwise disjoint copies of $(0,1)$ whose union is $Y \backslash Y_{0}$. We may again assume that every element of $\mathbf{J}_{0}$ has at least one endpoint in $Y_{0}$. Let $R_{0}$ be the set of midpoints of elements of $\mathbf{J}_{0}$. Let $\left\{R_{n}: 1 \leq n<\omega\right\}$ be the collection of cut-sets for $Y$ and set $R=\bigcup_{n>\omega} R_{n}$. Let $P_{n}$ be the component of $Y \backslash R_{0}$ that contains $y_{n}$. For each $0<n<\omega$ let $\mathbf{J}_{n}$ be the set of intervals of PR $[Y]$ derived from $R_{n}$, each indexed as before by the elements of $\Sigma$. Let $\left\{N_{k}: k<\omega\right\}$ be the collection of 
sets of elements of $\mathrm{PR}[Y]$ compatible with $\left\{\widehat{R}_{k}: k<\omega\right\}$ and let $\left\{T_{E}: E \in N\right\}$ be the fundamental partition of PR[Y] based on $N=\bigcup_{k<\omega} N_{k}$. If $E \subset Q$ and $f: E \rightarrow R$, then $f$ is level preserving if $f\left(E \cap Q_{n}\right) \subset R_{n}$ for all $n<\omega$.

For each $I \in \mathbf{I}_{n}$ and $J \in \mathbf{J}_{n}$ there is a unique linear homeomorphism between $I$ and $J$ that preserves orientation. Denote this homeomorphism by $\eta_{I, J}$. If $\sigma, \tau \in \Sigma(n), I=I_{\sigma \mid m+1}$, and $J=J_{\tau \mid m+1}$ for some $m<n$, then $\eta_{I, J}\left(I_{\sigma}\right)=J_{\tau}$ if and only if $\sigma(k)=\tau(k)$ for all $m<k \leq n$. If $\Gamma: \mathbf{I}_{n} \rightarrow \mathbf{J}_{n}$ is a bijection, then $\Gamma^{*}: \bigcup \mathbf{I}_{n} \rightarrow \bigcup \mathbf{J}_{n}$ is the function $\bigcup_{I \in I_{n}} \eta_{I, \Gamma(I)} . \Gamma^{*}$ is a homeomorphism that is linear and orientation preserving on each element of $\mathbf{I}_{n}$.

Now order each $\mathbf{I}_{n}$ and $\mathbf{J}_{n}$ lexicographically using the indices of their elements. These collections then have order-type $\omega^{2}$. Let $\mathbf{F} \subset \mathbf{I}_{n}$ and $\mathbf{G} \subset \mathbf{J}_{n}$ be equipotent finite sets and let $\gamma: F \rightarrow G$ be a bijection. Then $\mathbf{I}_{n} \backslash \mathbf{F}$ and $\mathbf{J}_{n} \backslash \mathbf{G}$ still have order-type $\omega^{2}$, so there is a unique order isomorphism $\Delta_{F}: \mathbf{I}_{n} \backslash F \rightarrow$ $\mathbf{J}_{n} \backslash G$. Define $\Gamma: \mathbf{I}_{n} \rightarrow \mathbf{J}_{n}$ by $\Gamma=\gamma \cup \Delta_{F}$. Then $\Gamma$ is a bijection.

In those situations where more than one $F$ is being considered and subscripts are used to distinguish the various set, the same subscripts will be used to distinguish the corresponding $\gamma, \Delta$, and $\Gamma$ functions. For example, the functions associated with $\mathbf{F}_{1}$ will be $\gamma_{1}, \Delta_{1}$, and $\Gamma_{1}$.

It will be necessary in what follows to compare the index of $I_{\sigma}$ with that of $\gamma\left(I_{\sigma}\right)$ or $\Gamma\left(I_{\sigma}\right)$. In order to facilitate this, we will use $\gamma(\sigma)$ and $\Gamma(\sigma)$ to denote the indices of $\gamma\left(I_{\sigma}\right)$ and $\Gamma\left(I_{\sigma}\right)$ respectively.

The next lemma is obvious and its proof is omitted.

Lemma 3. Let $m \leq n<\omega$ and let $\mathbf{F}_{1} \subset \mathbf{I}_{m}$ and $\mathbf{F}_{2} \subset \mathbf{I}_{n}$ with $\left\{I \in \mathbf{I}_{n}: I \subset\right.$ $\left.\mathbf{F}_{1}\right\} \subset \bigcup \mathbf{F}_{2}$. If $\gamma_{1}: F_{1} \rightarrow \mathbf{J}_{m}$ is a one-to-one function and $\gamma_{2}: \mathbf{F}_{2} \rightarrow \mathbf{J}_{n}$ is defined by $\gamma_{2}(I)=\Gamma_{1}^{*}(I)$, then $\Gamma_{1}^{*}(I)=\Gamma_{2}^{*}(I)$ for all $I \in \mathbf{I}_{n}$.

Lemma 4. Let $\mathbf{F} \subset \mathbf{I}_{k}$ be finite and let $\gamma: \mathbf{F} \rightarrow \mathbf{J}_{k}$ be a one-to-one function. Assume that there are $b, c, m<\omega$ such that

1. $c-m>b$;

2. if $I_{\sigma} \in \mathbf{F}$ then either $\sigma(1) \leq b$ or $\sigma(1)>c$;

3. if $I_{\sigma} \in \mathbf{F} \cap \mathbf{I}_{k, n}$ and $m \leq \sigma(1) \leq b$ then $\gamma\left(I_{\sigma}\right) \in \mathbf{J}_{k, n}$ and $\gamma(\sigma)(1) \leq b$; and

4. if $I_{\sigma} \in \mathbf{F} \cap \mathbf{I}_{k, n}$ and $\sigma(1)>c$ then $\gamma\left(I_{\sigma}\right) \in \mathbf{J}_{k, n}$ and $\gamma(\sigma)(1)>b$. Then $\Gamma\left(I_{\sigma}\right) \in \mathbf{J}_{k, n}$ and $\Gamma(\sigma)(1)>b$ for all $I_{\sigma} \in \mathbf{I}_{k, n}$ with $\sigma(1)>c$.

Proof. Let $n<\omega$. The elements of $\mathbf{J}_{k, n} \backslash \gamma(\mathbf{F})$ are the images under $\Delta_{F}$ of $\mathbf{I}_{k, n} \backslash \mathbf{F}$. By conditions 2 and 3 ,

$$
\begin{aligned}
\left|\mathbf{F} \cap\left\{I_{\sigma} \in \mathbf{I}_{k, n}: m \leq \sigma(1) \leq c\right\}\right| & =\left|\mathbf{F} \cap\left\{I_{\sigma} \in \mathbf{I}_{k, n}: m \leq \sigma(1) \leq b\right\}\right| \\
& =\mid\left\{\gamma\left(I_{\sigma}\right): I_{\sigma} \in \mathbf{I}_{k, n} \text { and } m \leq \sigma(1) \leq b\right\} \mid \\
& \leq \mid\left\{J_{\sigma} \in \mathbf{J}_{k, n}: J_{\sigma} \in \gamma(F) \text { and } \sigma(1) \leq b\right\} \mid \\
& =\left|\gamma(\mathbf{F}) \cap\left\{J_{\sigma} \in \mathbf{J}_{k, n}: \sigma(1) \leq b\right\}\right| .
\end{aligned}
$$


Also, $\left|\left\{I_{\sigma} \in \mathbf{I}_{k, n}: m \leq \sigma(1) \leq c\right\}\right| \geq\left|\left\{J_{\sigma} \in \mathbf{J}_{k, n}: \sigma(1) \leq b\right\}\right|$ because $c-m>b$. Therefore,

$$
\begin{aligned}
\mid\left\{I_{\sigma}\right. & \left.\in \mathbf{I}_{k, n}: m \leq \sigma(1) \leq c\right\} \backslash \mathbf{F} \mid \\
& =\left|\left\{I_{\sigma} \in \mathbf{I}_{k, n}: m \leq \sigma(1) \leq c\right\} \backslash\left(\mathbf{F} \cap\left\{I_{\sigma} \in \mathbf{I}_{k, n}: m \leq \sigma(1) \leq c\right\}\right)\right| \\
& \geq\left|\left\{J_{\sigma} \in \mathbf{J}_{k, n}: \sigma(1) \leq b\right\} \backslash\left(\gamma(\mathbf{F}) \cap\left\{J_{\sigma} \in \mathbf{J}_{k, n}: \sigma(1) \leq b\right\}\right)\right| \\
& =\left|\left\{J_{\sigma} \in \mathbf{J}_{k, n}: \sigma(1) \leq b\right\} \backslash \gamma(\mathbf{F})\right| .
\end{aligned}
$$

Thus, if $J_{\tau} \in \mathbf{J}_{k, n}$ and $\tau(1) \leq b$ then there is $I_{\sigma} \in \mathbf{I}_{k}$ such that either $I_{\sigma} \in \mathbf{F}$ or $I_{\sigma} \in \mathbf{I}_{k, n}$ and $\sigma(1) \leq c$, and $\Gamma\left(I_{\sigma}\right)=\mathbf{J}_{\tau}$. It follows from this and condition 4 that if $I_{\sigma} \in \mathbf{I}_{k, n}$ and $\sigma(1)>c$, then $\Gamma\left(I_{\sigma}\right) \in \mathbf{J}_{k, n}$ and $\Gamma(\sigma)(1)>b$.

Lemma 5. Let $\mathbf{F}_{1}, \mathbf{F}_{2} \subset I_{k}$ be finite and let $\gamma_{1}: \mathbf{F}_{1} \rightarrow \mathbf{J}_{k}$ and $\gamma_{2}: \mathbf{F}_{2} \rightarrow \mathbf{J}_{k}$ be one-to-one functions. Let $a, b, m<\omega$ such that

1. $b-a>m$

2. $\left\{I_{\sigma} \in \mathbf{F}_{1}: \sigma(1) \leq a\right\}=\left\{I_{\sigma} \in \mathbf{F}_{2}: \sigma(1) \leq a\right\}=\mathbf{G}$; and

3. $\gamma_{1}\left(I_{\sigma}\right)=\gamma_{2}\left(I_{\sigma}\right)$ for all $I_{\sigma} \in \mathbf{G}$;

and that for $i=1$ or 2 ,

4. if $J_{\sigma} \in \gamma_{i}\left(\mathbf{F}_{i}\right)$ then either $\sigma(1) \leq a$ or $\sigma(1)>b$;

5. if $I_{\sigma} \in \mathbf{F}_{i}$ and $\sigma(1)>b$ then $\gamma_{i}(\sigma)(1)>a$; and

6. for all $n<\omega$, if $J_{\sigma} \in \gamma_{i}\left(\mathbf{F}_{i}\right) \cap \mathbf{J}_{k, n}$ and $\gamma_{i}^{-1}\left(J_{\sigma}\right) \notin \mathbf{I}_{k, n}$ then $\sigma(1)<m$. Then $\Gamma_{1}\left(I_{\sigma}\right)=\Gamma_{2}\left(I_{\sigma}\right)$ for all $I_{\sigma} \in I_{n}$ with $\sigma(1) \leq a$.

Proof. Let $n<\omega$. By condition 2,

$$
\left\{I_{\sigma} \in \mathbf{I}_{k, n}: \sigma(1) \leq a\right\} \cap \mathbf{F}_{1}=\mathbf{I}_{k, n} \cap \mathbf{G}=\left\{I_{\sigma} \in \mathbf{I}_{k, n}: \sigma(1) \leq a\right\} \cap \mathbf{F}_{2}
$$

and

$$
\begin{aligned}
\left\{I_{\sigma} \in \mathbf{I}_{k, n}: \sigma(1) \leq a\right\} \backslash \mathbf{F}_{1} & =\left\{I_{\sigma} \in \mathbf{I}_{k, n}: \sigma(1) \leq a\right\} \backslash \mathbf{G} \\
& =\left\{I_{\sigma} \in \mathbf{I}_{k, n}: \sigma(1) \leq a\right\} \backslash \mathbf{F}_{2} .
\end{aligned}
$$

By conditions 2, 3, and 4,

$$
\begin{aligned}
\left\{J_{\sigma} \in \mathbf{J}_{k, n}: \sigma(1) \leq b\right\} \backslash \gamma_{1}\left(\mathbf{F}_{1}\right) & =\left\{J_{\sigma} \in \mathbf{J}_{k, n}: \sigma(1) \leq b\right\} \backslash \gamma_{1}(\mathbf{G}) \\
& =\left\{J_{\sigma} \in \mathbf{J}_{k, n}: \sigma(1) \leq b\right\} \backslash \gamma_{2}\left(\mathbf{F}_{2}\right) .
\end{aligned}
$$

If $I_{\sigma} \in \mathbf{I}_{k, n} \cap \mathbf{G}$ then $\Gamma_{1}\left(I_{\sigma}\right)=\gamma_{1}\left(I_{\sigma}\right)=\gamma_{2}\left(I_{\sigma}\right)=\Gamma_{2}\left(I_{\sigma}\right)$. The values of $\Gamma_{1}$ and $\Gamma_{2}$ on $\left\{I_{\sigma} \in \mathbf{I}_{k, n}: \sigma(1) \leq a\right\} \backslash \mathbf{G}$ are determined by $\Delta_{1}$ and $\Delta_{2}$ respectively. We can establish the equality of $\Gamma_{1}$ and $\Gamma_{2}$ on $\left\{I_{\sigma} \in \mathbf{I}_{k, n}: \sigma(1) \leq a\right\} \backslash \mathbf{G}$ by showing that this set is no larger than $\left\{J_{\sigma} \in \mathbf{J}_{k, n}: \sigma(1) \leq b\right\} \backslash \gamma_{1}(\mathbf{G})$. Then, since both $\Delta_{1}$ and $\Delta_{2}$ take the $\alpha$ th element of $\left\{I_{\sigma} \in \mathbf{I}_{k, n}: \sigma(1) \leq a\right\} \backslash \mathbf{G}$ to the 
$\alpha$ th element of $\left\{J_{\sigma} \in \mathbf{G} J_{k, n}: \sigma(1) \leq b\right\} \backslash \gamma_{1}(\mathbf{G})$, they must be equal.

$$
\begin{aligned}
\mid\left\{J_{\sigma} \in \mathbf{J}_{k, n}:\right. & \sigma(1) \leq b\} \backslash \gamma_{1}(\mathbf{G}) \mid \\
= & \left|\left\{J_{\sigma} \in \mathbf{J}_{k, n}: \sigma(1) \leq a\right\} \backslash \gamma_{1}(\mathbf{G})\right|+\left|\left\{J_{\sigma} \in \mathbf{J}_{k, n}: a<\sigma(1) \leq b\right\}\right| \\
= & \mid\left\{J_{\sigma} \in \mathbf{J}_{k, n}: \sigma(1) \leq a\right\} \backslash\left(\left\{\gamma_{1}\left(I_{\sigma}\right) \in \mathbf{J}_{k, n}: I_{\sigma} \in \mathbf{G} \backslash \mathbf{I}_{k, n}\right\}\right. \\
& \left.\cup\left\{\gamma_{1}\left(I_{\sigma}\right) \in \mathbf{J}_{k, n}: I_{\sigma} \in \mathbf{G} \cap \mathbf{I}_{k, n}\right\}\right) \mid \\
& +\left|\left\{J_{\sigma} \in \mathbf{J}_{k, n}: a<\sigma(1) \leq b\right\}\right| \\
\geq & \left|\left\{J_{\sigma} \in \mathbf{J}_{k, n}: \sigma(1) \leq a\right\} \backslash\left\{\gamma_{1}\left(I_{\sigma}\right) \in \mathbf{J}_{k, n}: I_{\sigma} \in \mathbf{G} \cap \mathbf{I}_{k, n}\right\}\right| \\
= & \left|\left\{I_{\sigma} \in \mathbf{I}_{k, n}: \sigma(1) \leq a\right\} \backslash\left\{I_{\sigma} \in \mathbf{G} \cap \mathbf{I}_{k, n}: \gamma_{1}\left(I_{\sigma}\right) \in \mathbf{J}_{k, n}\right\}\right| \\
\geq & \left|\left\{I_{\sigma} \in \mathbf{I}_{k, n}: \sigma(1) \leq a\right\} \backslash \mathbf{G}\right| .
\end{aligned}
$$

\section{Proof OF TheOREM 1}

Let $X$ and $Y$ be $\omega$-graphs with dividing sets $X_{0}$ and $Y_{0}$. We will use the structures and definitions developed in $\S$ II. Let $g: Q_{1} \rightarrow R_{1}$ be a bijection such that $g\left(Q_{1} \cap O_{n}\right)=R_{1} \cap P_{n}$ for all $n<\omega$. Then $g\left(Q_{0}\right)=R_{0}$. For our convenience later in the proof, we will assume that the first $\mu(n)$ elements of any $\mathbf{I}_{m, n}$ are those elements of $\mathbf{I}_{m, n}$ having an element of $Q_{0}$ as an endpoint.

The homeomorphism we will define is essentially that defined by Norden in [N].

Define $\Gamma_{\phi}: \mathbf{I}_{1} \rightarrow \mathbf{J}_{1}$ by $\Gamma_{\phi}\left(I_{\sigma}\right)=J_{\sigma}$, and $h_{\phi}: \bigcup \mathbf{I}_{1} \rightarrow \bigcup \mathbf{J}_{1}$ by $h_{\phi}=\Gamma_{\phi}^{*}$. Then $h_{\phi}$ is a homeomorphism. Set $\theta(\phi)=\phi$.

Let $E \in M_{1}$. Set $f_{E}=g \mid E$ and $\theta(E)=f_{E}(E)$. Let $\mathbf{F}_{E}=\widehat{\mathbf{F}}_{E}$ and $\mathbf{F}_{\theta(E)}=\widehat{\mathbf{F}}_{\theta(E)}$. Each $I \in \mathbf{F}_{E}$ is adjacent to exactly one element of $E$ and each element of $E$ is the endpoint of exactly two elements of $F_{E}$. Similarly, each element of $F_{\theta(E)}$ is adjacent to exactly one element of $\theta(E)$ and each element of $\theta(E)$ is the endpoint of exactly two elements of $F_{\theta(E)}$. Define $\gamma_{E}: \mathbf{F}_{E} \rightarrow \mathbf{F}_{\theta(E)}$ as follows. Let $I \in \mathbf{F}_{E}$ and let $p \in E$ be an endpoint of $I$. If $p$ is the right-hand endpoint of $I$, then set $\gamma_{E}(I)$ equal to the element of $\mathbf{F}_{\theta(E)}$ which has $g(p)$ for its right-hand endpoint. If $p$ is the left-hand endpoint of $I$, then set $\gamma_{E}(I)$ equal to the element of $\mathbf{F}_{\theta(E)}$ which has $g(p)$ for its lefthand endpoint. Then $\gamma_{E}$ is a bijection. Define $h_{E}:\left(\cup \mathbf{I}_{2}\right) \cup E \rightarrow\left(\cup \mathbf{J}_{2}\right) \cup \theta(E)$ by $h_{E}=\Gamma_{E}^{*} \cup f_{E}$. Both $\Gamma_{E}^{*}$ and $f_{E}$ are bijections so $h_{E}$ is a bijection. It is also a homeomorphism on $U I_{2}$ because $\Gamma_{E}^{*}$ is. Let $x \in E$ and let $V$ be a neighborhood of $f_{E}(x)$ in $Y$. By the definition of $\gamma_{E}$ there is a neighborhood $U$ of $x$ in $A^{*}(x) \cup\{x\}$ such that $h_{E}(U) \subset V$. Thus $h_{E}$ is continuous at $x$. A similar argument shows that $h_{E}^{-1}$ is continuous at $h_{E}(x)$, so $h_{E}$ is a homemorphism. 
Let $2 \leq 1<\omega$ and assume that for all $k<1$ and all $E \in M_{k}$,

1. $f_{E}: E \rightarrow \widehat{R}_{k}$ is a level preserving one-to-one function and $\theta(E)=$ $f_{E}(E)$

2. $\mathbf{F}_{E} \subset \mathbf{I}_{k+1}$ and $\mathbf{F}_{\theta(E)} \subset \mathbf{J}_{k+1}$ are finite and $\gamma_{E}: \mathbf{F}_{E} \rightarrow \mathbf{F}_{\theta(E)}$ is a bijection; and

3. the function $h_{E}:\left(\cup \mathbf{I}_{k+1}\right) \cup E \rightarrow\left(\cup \mathbf{J}_{k+1}\right) \cup \theta(E)$ given by $h_{E}=\Gamma_{E}^{*} \cup f_{E}$ is a homeomorphism.

Fix $E \in M_{l}$. Each element of $E \cap Q_{l}$ is the midpoint of some element of $I_{l-1}$ and $h_{E^{\prime \prime}}$, which is defined on $\bigcup I_{l-1}$, takes midpoints to midpoints. Thus $h_{E^{\prime \prime}}(p) \in R_{l}$ for all $p \in E \cap Q_{l}$. Define $f_{E}: E \rightarrow \widehat{R}_{l}$ by

$$
f_{E}(p)= \begin{cases}h_{E^{\prime}}(p) & \text { if } p \in E \cap \widehat{Q}_{l-1}, \\ h_{E^{\prime \prime}}(p) & \text { if } p \in E \cap Q_{l} .\end{cases}
$$

Then $f_{E}$ is a one-to-one level preserving function. Note that if $p \in E \cap \widehat{Q}_{l-1}$ then $f_{E}(p)=h_{E^{\prime}}(p)=f_{E^{\prime}}(p)$. Extending this backward, we can see that if $1 \leq k<l$ and $p \in E \cap \widehat{Q}_{k}$ then $f_{E}(p)=f_{E \cap \widehat{Q}_{k}}(p)$.

Let $\mathbf{F}_{E 1}=\mathbf{A}\left(E \cap Q_{l}\right)$ and $\mathbf{F}_{\theta(E) 1}=\mathbf{A}\left(\theta(E) \cap R_{l}\right)$. Let $I \in \mathbf{F}_{E 1}$ and let $p \in E \cap Q_{l}$ be and endpoint of $I$. Then $f_{E}(p)=h_{E^{\prime \prime}}(p) \in R_{l}$ and $h_{E^{\prime \prime}}(p)$ is an endpoint of $h_{E^{\prime \prime}}(I)$ because $h_{E^{\prime \prime}}$ is continuous. Thus $h_{E^{\prime \prime}}(I) \in \mathbf{F}_{\theta(E) 1}$. A similar argument shows that if $h_{E^{\prime \prime}}(I) \in \mathbf{F}_{\theta(E) 1}$ then $I \in \mathbf{F}_{E 1}$.

Let $\mathbf{F}_{E 2}=\left\{I \in \widehat{\mathbf{F}}_{E} \backslash \mathbf{F}_{E 1}: h_{E^{\prime}}(I) \in \widehat{\mathbf{F}}_{\theta(E)} \backslash \mathbf{F}_{\theta(E) 1}\right\}$ and let $\mathbf{F}_{\theta(E) 2}=\{J \in$ $\left.\widehat{\mathbf{F}}_{\theta(E)} \backslash \mathbf{F}_{\theta(E) 1}: h_{E^{\prime}}^{-1}(J) \in \widehat{\mathbf{F}}_{E} \backslash \mathbf{F}_{E 1}\right\}$. Clearly $I \in \mathbf{F}_{E 2}$ if and only if $h_{E^{\prime}}(\mathbf{I}) \in$ $\mathbf{F}_{\theta(E) 2}$. Set $\mathbf{F}_{E}=\mathbf{F}_{E 1} \cup \mathbf{F}_{E 2}$ and $\mathbf{F}_{\theta(E)}=\mathbf{F}_{\theta(E) 1} \cup \mathbf{F}_{\theta(E) 2}$. Define $\gamma_{E}: \mathbf{F}_{E} \rightarrow \mathbf{F}_{\theta(E)}$ by

$$
\gamma_{E}(I)= \begin{cases}h_{E^{\prime \prime}}(I) & \text { if } I \in \mathbf{F}_{E 1}, \\ h_{E^{\prime}}(I) & \text { if } I \in \mathbf{F}_{E 2} .\end{cases}
$$

Then $\gamma_{E}$ is a bijection.

Define $h_{E}:\left(\cup \mathbf{I}_{l+1}\right) \cup E \rightarrow\left(\cup \mathbf{J}_{l+1}\right) \cup \theta(E)$ by $h_{E}=\Gamma_{E}^{*} \cup f_{E}$. The function $h_{E}$ is a bijection because $\Gamma_{E}^{*}$ and $f_{E}$ are bijections and is a homemorphism on $\cup \mathbf{I}_{l+1}$ because $\Gamma_{E}^{*}$ is. If $p \in E \cap Q_{l}$ then $\mathbf{A}(p) \subset \mathbf{F}_{E 1}$ and $h_{E}\left(\mathbf{A}^{*}(p) \cup\{p\}\right)=$ $h_{E^{\prime \prime}}\left(\mathbf{A}^{*}(p) \cup\{p\}\right)$. Now let $p \in E^{\prime}$. If $I \in \mathbf{A}_{l+1}(p)$ then $I \in \widehat{\mathbf{F}}_{E}$. Since $p$ is an endpoint of $I$ and $p \in \widehat{Q}_{l-1}$, the other endpoint of $I$ must be an element of $Q_{l+1}$. Hence $I \notin \mathbf{F}_{E 1}$. To show that $h_{E^{\prime}}(I) \in \widehat{\mathbf{F}}_{\theta(E)} \backslash \mathbf{F}_{\theta(E) 1}$, note that $p \in E^{\prime}$ and $h_{E^{\prime}}$ is continuous on $\left(\cup I_{l}\right) \cup E^{\prime}$. So $f_{E}(p)=\mathbf{F}_{E^{\prime}}(p)$ is an endpoint of $h_{E^{\prime}}(I)$. But $f_{E^{\prime}}$ is level preserving, so $f_{E^{\prime}}(p) \in \widehat{R}_{l+1}$. Again, the other endpoint of $h_{E^{\prime}}(I)$ must be an element of $R_{l+1}$. Hence $h_{E^{\prime}}(I) \in \widehat{\mathbf{F}}_{\theta(E)} \backslash \mathbf{F}_{\theta(E) 1}$. It follows that $\mathbf{A}_{l+1}(p) \subset \mathbf{F}_{E 2}$ and $h_{E}\left(\mathbf{A}_{l+1}^{*}(p) \cup\{p\}\right)=h_{E^{\prime}}\left(\mathbf{A}_{l+1}^{*}(p) \cup\{p\}\right)$. But $h_{E^{\prime}}$ is a homeomorphism on $\left(\cup \mathbf{I}_{l}\right) \cup E^{\prime}$ and $h_{E^{\prime \prime}}$ is a homeomorphism on $\cup \mathbf{I}_{l-1}$, so $h_{E}$ is a homeomorphism on $\left(\cup \mathbf{I}_{l+1}\right) \cup E$. 
Notice that for any $k<\omega, E \in M_{k}, x_{n} \in X_{0}$, and $I_{\sigma} \in \mathbf{I}_{k, n}$, if $\Gamma_{E}\left(I_{\sigma}\right) \notin$ $\mathbf{J}_{k, n}$ then $\sigma(1)<\mu(n)$ because only the first $\mu(n)$ elements of $\mathbf{I}_{1, n}$ have endpoints in $Q_{0}$.

For all $n<\omega$ and all $E \in M_{n}$, define $H_{E}: S_{E} \rightarrow T_{\theta(E)}$ by $H_{E}(A)=$ $\lambda\left(A \cap X_{0}\right) \cup h_{E}\left(A \backslash X_{0}\right)$. Finally, define $H: \operatorname{PR}[X] \rightarrow \operatorname{PR}[Y]$ by $H=\bigcup_{E \in M} H_{E}$. To show that $H$ is a bijection it is sufficient to show that $\theta$ is a bijection. Let $E, D \in M$ and $E \neq D$. Then $\theta(E)=f_{E}(E)$ and $\theta(D)=f_{D}(D)$. Both $f_{E}$ and $f_{D}$ are level-preserving one-to-one functions, so $\theta(E) \neq \theta(D)$ if $E \in M_{k}$ and $D \in M_{l}$ and $k \neq l$. Assume that $E, D \in M_{1}$. Then $\theta(E)=g(E) \neq g(D)=$ $\theta(D)$ since $g$ is a bijection. Assume that $E, D \in M_{k}$ for some $k>1$. Either $E \cap Q_{k} \neq D \cap Q_{k}$ or $E^{\prime} \neq D^{\prime}$. But the functions $h_{E^{\prime}}, h_{E^{\prime \prime}}, h_{D^{\prime}}$, and $h_{D^{\prime \prime}}$ are all one-to-one, so either $h_{E^{\prime \prime}}\left(E \cap Q_{k}\right) \neq h_{D^{\prime \prime}}\left(D \cap Q_{k}\right)$ or $h_{E^{\prime}}\left(E^{\prime}\right) \neq h_{D^{\prime}}\left(D^{\prime}\right)$. In either case, $\theta(E) \neq \theta(D)$.

Let $A \in S_{E}$ where $E \in M_{k}$ and let $V$ be a neighborhood of $H(A)$ in $Y$. Pick $a<\omega$ such that if $I_{\sigma} \in \mathbf{A}_{1}(A)$ then $\sigma(1) \leq a$ and if $J_{\sigma} \in \mathbf{A}_{1}(H(A))$ then $\sigma(1) \leq a$. Let $m=\max \left\{\mu(n): \mathbf{A}_{1}(A) \cap \mathbf{I}_{1, n} \neq \varnothing\right.$ or $\left.\mathbf{A}_{1}(H(A)) \cap \mathbf{J}_{1, n} \neq \varnothing\right\}+1$. Pick $b \in \omega$ such that $b-m>a$ and

$$
\text { Int }\left[\mathrm{Cl}\left(\bigcup\left\{J_{\sigma} \in \mathbf{J}_{1, n}: \sigma(1)>b\right\}\right)\right] \subset V
$$

for all $y_{n} \in H(A) \cap Y_{0}$. Set

$$
V_{y_{n}}=\operatorname{Int}\left[\mathrm{Cl}\left(\bigcup\left\{J_{\sigma} \in \mathbf{J}_{1, n}: \sigma(1)>b\right\}\right)\right]
$$

and set $V_{0}=\bigcup_{p \in H(A) \cap Y_{0}} V_{p}$. Pick $c \in \omega$ such that $c-m>b$ and if $x_{n} \in A \cap X_{0}$ and $p \in Q_{1} \cap \operatorname{Int}\left[\mathrm{Cl}\left(\bigcup\left\{I_{\sigma} \in \mathbf{I}_{1, n}: \sigma(1)>c\right\}\right)\right]$, then $g(p) \in V_{y_{n}}$. For each $x_{n} \in A \cap X_{0}$ set $U_{x_{n}}=\operatorname{Int}\left[\mathrm{Cl}\left(\bigcup\left\{I_{\sigma} \in \mathbf{I}_{1, n}: \sigma(1)>c\right\}\right)\right]$. Let $U_{0}=\bigcup_{p \in A \cap X_{0}} U_{p}$. If $A \cap X_{0}=\varnothing$ then set $U_{0}=\varnothing$. Pick $r \geq k+1$ such that $h_{E}\left(\mathbf{A}_{r}^{*}(p)\right) \subset V$ for all $p \in A \backslash X_{0}$. Set $U_{p}=\mathbf{A}_{r}^{*}(p) \cup\{p\}$ for $p \in A \backslash X_{0}$ and set $U_{1}=\bigcup_{p \in A \backslash X_{0}} U_{p}$. Let $U=U_{0} \cup U_{1}$. Note that:

1. if $I_{\sigma} \cap U_{1} \neq \varnothing$ then $\sigma(1) \leq a$;

2. if $\bar{J}_{\sigma} \cap\left(H(A) \backslash Y_{0}\right) \neq \varnothing$ then $\sigma(1) \leq a$;

3. if $I_{\sigma} \cap U_{x_{n}} \neq \varnothing$ for some $x_{n} \in A \cap X_{0}$ then $I_{\sigma\lceil 1} \in \mathbf{I}_{1, n}$ and $\sigma(1)>c$;

4. if $J_{\sigma} \cap V_{y_{n}} \neq \varnothing$ for some $y_{n} \in H(A) \cap Y_{0}$ then $J_{\sigma \mid 1} \in \mathbf{J}_{1, n}$ and $\sigma(1)>b$;

5. if $p \in A \backslash X_{0}$ then $U_{p} \cap \widehat{Q}_{k+1} \subset\{p\}$.

6. $a, b, c$ and $m$ satisfy condition 1 in Lemmas 4 and 5; and

7. if $I_{\sigma} \in \mathbf{I}_{1, n}$ and $m \leq \sigma(1)$ then $H_{D}\left(I_{\sigma}\right) \subset \bigcup \mathbf{J}_{1, n}$ for any $0<1<$ $\omega, n<\omega$, and $D \in M$.

The heart of the proof that $H([A, U]) \subset[H(A), V]$ is contained in Lemmas 6 and 7.

Lemma 6. Let $D \in M_{j}$ where $1 \leq j \leq k, D \subset U$, and $D \cap U_{1}=E \cap \widehat{Q}_{j}$. Let $C=E \cap \widehat{Q}_{j}$. Then

1. if $p \in D \cap U_{q}$ for some $q \in A \cap X_{0}$ then $f_{D}(p) \in V_{\lambda(q)}$; 
2. if $p \in D \cap U_{q}$ for some $q \in E$ then $p=q$ and $f_{D}(p)=F_{E}(p)$;

3. if $I_{\sigma} \in \mathbf{I}_{j+1}$ and $\sigma(1) \leq a$ then $\Gamma_{C}\left(I_{\sigma}\right)=\Gamma_{D}\left(I_{\sigma}\right)$; and

4. if $I_{\sigma} \in \mathbf{I}_{j+1, n}, x_{n} \in A \cap X_{0}$, and $\sigma(1)>c$, then $\Gamma_{D}\left(I_{\sigma}\right) \in \mathbf{J}_{j+1, n}$ and $\Gamma_{D}(\sigma)(1)>b$.

Proof. To begin with, let us take note of three useful facts. First, since $\Gamma_{\phi}\left(I_{\sigma}\right)=$ $J_{\sigma}$ for all $I_{\sigma} \in I_{1}$, if $I_{\sigma} \in I_{1, n}$ and $\sigma(1)>c$, then $\Gamma_{\phi}\left(I_{\sigma}\right)=J_{\sigma} \in \mathbf{J}_{1, n}$ and $\Gamma_{\phi}(\sigma)(1)=\sigma(1)>c>b$. Also, for any $j$, if $p \in C$ then $f_{C}(p)=f_{E}(p)$. Furthermore, if $I_{\sigma} \in F_{D}$ then either $\sigma(1) \leq a<b$ or $\sigma(1)>c$.

Let $j=1$. Then $D \subset Q_{1}$ and $D \cap U_{1}=E \cap Q_{1}$. Let $p \in D$. If $p \in U_{q}$ for some $q \in A \cap X_{0}$, then $f_{D}(p)=g(p) \in V_{\lambda(q)}$. If $p \in U_{q}$ for some $q \in A \backslash X_{0}$, then $q \in E, p=q$, and $f_{D}(p)=g(p)=f_{C}(p)$.

Let $n<\omega$ and let $I_{\sigma} \in \mathbf{I}_{2, n} \cap \mathbf{F}_{D}$ with $\sigma(1)>c$. Let $p \in D$ be an endpoint of $I_{\sigma}$. Since $\sigma(1)>c, p$ must be in $U_{x_{n}}$. Then $f_{D}(p)$, which is an endpoint of $\gamma_{D}\left(I_{\sigma}\right)$, is in $V_{y_{n}}$. Thus $\gamma_{D}\left(I_{\sigma}\right) \in \mathbf{J}_{2, n}$ and $\gamma_{D}(\sigma)(1)>b>a$.

It follows from $D \cap U_{1}=C$ that $F_{C}=\left\{I_{\sigma} \in \mathbf{F}_{D}: \sigma(1) \leq a\right\}$. Let $I_{\sigma} \in \mathbf{F}_{C}$. Let $p \in D$ be an endpoint of $I_{\sigma}$. Then $p$ must be an element of $U_{1}$, so $f_{D}(p)=$ $f_{E}(p)=f_{C}(p)$. Thus $f_{E}(p)$ is an endpoint for both $\gamma_{C}\left(I_{\sigma}\right)$ and $\gamma_{D}\left(I_{\sigma}\right)$. Since both $\gamma_{C}$ and $\gamma_{D}$ preserve orientation, it must be true that $\gamma_{C}\left(I_{\sigma}\right)=\gamma_{D}\left(I_{\sigma}\right)$. Also, $\gamma_{D}(\sigma)(1) \leq a<b$ because $f_{D}(p) \in H(A) \backslash Y_{0}$.

By Lemma 4, if $I_{\sigma} \in \mathbf{I}_{2, n}$ and $\sigma(1)>c$, then $\Gamma_{D}\left(I_{\sigma}\right) \in \mathbf{J}_{2, n}$ and $\Gamma(\sigma(1))>$ $b$. By Lemma 5, if $I_{\sigma} \in \mathbf{I}_{2}$ and $\sigma(1) \leq a$, then $\Gamma_{D}\left(I_{\sigma}\right)=\Gamma_{C}\left(I_{\sigma}\right)$.

Let $2 \leq j \leq k$ and assume that the lemma is valid for all $1 \leq i<j$ and all $D \in M_{i}$ with $D \subset U$ and $D \cap U_{1}=E \cap \widehat{Q}_{i}$. Let $D \in M_{j}$ with $D \subset U$ and $D \cap U_{1}=E \cap \widehat{Q}_{j}$. Then $D^{\prime} \in M_{j-1}, D^{\prime} \subset U$, and $D^{\prime} \cap U_{1}=E \cap \widehat{Q}_{j-1}=C^{\prime}$, so the lemma is valid for $D^{\prime}$. If $j=2$, then $D^{\prime \prime}=C^{\prime \prime}=\varnothing$. If $j>2$, then $D^{\prime \prime} \in M_{j-2}, D^{\prime \prime} \subset U$, and $D^{\prime \prime} \cap U_{1}=E \cap \widehat{Q}_{j-2}=C^{\prime \prime}$. Thus the lemma is valid for $D^{\prime \prime}$.

Let $p \in D \cap U_{x_{n}}$ for some $x_{n} \in A \cap X_{0}$. If $p \in \widehat{Q}_{j-1}$ then $f_{D}(p)=f_{D^{\prime}}(p) \in$ $V_{y_{n}}$. If $p \in Q_{j}$ then $f_{D}(p)=h_{D^{\prime \prime}}(p)$. Now $p$ is the midpoint of some element $I_{\sigma}$ of $I_{j-1, n}$ where $\sigma(1)>c$. But $\Gamma_{D^{\prime \prime}}\left(I_{\sigma}\right) \in J_{j-1, n}, \Gamma_{D^{\prime \prime}}(\sigma)(1)>b$, and $h_{D^{\prime \prime}}(p)$ is the midpoint of $\Gamma_{D^{\prime \prime}}\left(I_{\sigma}\right)$. Hence $f_{D}(p) \in V_{y_{n}}$.

Let $p \in D \cap U_{q}$ for some $q \in A \backslash X_{0}$. Then $q \in E$ and $q=p$. If $p \in \widehat{Q}_{j-1}$ then $f_{D}(p)=f_{d^{\prime}}(p)=f_{E}(p)$. If $p \in Q_{j}$ then

$$
f_{D}(p)=h_{D^{\prime \prime}}(p)=\Gamma_{D^{\prime \prime}}^{*}(p)=\Gamma_{C^{\prime \prime}}^{*}(p)=h_{C^{\prime \prime}}(p)=f_{C}(p)=f_{E}(p) .
$$

Let $n<\omega$ and let $I_{\sigma} \in F_{D} \cap \mathbf{I}_{j+1, n}$ with $\sigma(1)>c$. Either $\gamma_{D}\left(I_{\sigma}\right)=\Gamma_{D^{\prime}}^{*}\left(I_{\sigma}\right)$ or $\gamma_{D}\left(I_{\sigma}\right)=\Gamma_{D^{\prime \prime}}^{*}\left(I_{\sigma}\right)$. In either case, $\gamma_{D}\left(I_{\sigma}\right) \in \mathbf{J}_{j+1, n}$ and $\gamma_{D}(\sigma)(1)>b>a$.

It follows from the inductive hypotheses that $\mathbf{F}_{C 1}=\left\{I_{\sigma} \in \mathbf{F}_{D 1}: \sigma(1) \leq a\right\}$ and $\mathbf{F}_{C 2}=\left\{I_{\sigma} \in \mathbf{F}_{D 2}: \sigma(1) \leq a\right\}$. Thus $\mathbf{F}_{C}=\left\{I_{\sigma} \in \mathbf{F}_{D}: \sigma(1) \leq a\right\}$. Let $I_{\sigma} \in \mathbf{F}_{C}$. If $I_{\sigma} \in \mathbf{F}_{D 1}$ then $\gamma_{D}\left(I_{\sigma}\right)=\Gamma_{D^{\prime \prime}}^{*}\left(I_{\sigma}\right)$. But $\Gamma_{D^{\prime \prime}}^{*}\left(I_{\sigma}\right)=\Gamma_{C^{\prime \prime}}^{*}\left(I_{\sigma}\right)$ so $\gamma_{D}\left(I_{\sigma}\right)=\gamma_{C}\left(I_{\sigma}\right)$. If $I_{\sigma} \in \mathbf{F}_{D 2}$ then $\gamma_{D}\left(I_{\sigma}\right)=\Gamma_{D^{\prime}}^{*}\left(I_{\sigma}\right)$. But $\Gamma_{D^{\prime}}^{*}\left(I_{\sigma}\right)=\Gamma_{C^{\prime}}^{*}\left(I_{\sigma}\right)$ so $\gamma_{D}\left(I_{\sigma}\right)=\gamma_{C}\left(I_{\sigma}\right)$. In either case, $\gamma_{D}(\sigma)(1) \leq a<b$. 
By Lemma 4, if $I_{\sigma} \in \mathbf{I}_{j+1, n}$ and $\sigma(1)>c$, then $\Gamma_{D}\left(I_{\sigma}\right) \in \mathbf{J}_{j+1, n}$ and $\Gamma_{D}(\sigma)(1)>b$. By Lemma 5 , if $I_{\sigma} \in \mathbf{I}_{j+1}$ and $\sigma(1) \leq a$, then $\Gamma_{D}\left(I_{\sigma}\right)=\Gamma_{C}\left(I_{\sigma}\right)$.

Lemma 7. If $k \leq l, D \in M_{l}$, and $E \subset D \subset U$, then

1. if $p \in D \cap U_{q}$ for some $q \in A \cap X_{0}$ then $f_{D}(p) \in V_{\lambda(q)}$;

2. if $p \in D \cap U_{q}$ for some $q \in A \backslash X_{0}$ then $f_{D}(p) \in V$;

3. if $I_{\sigma} \in \mathbf{I}_{l+1, n}$ for some $x_{n} \in A \cap X_{0}$ and $\sigma(1)>c$, then $\Gamma_{D}\left(I_{\sigma}\right) \in \mathbf{J}_{l+1, n}$ and $\Gamma_{D}(\sigma)(1)>b$; and

4. if $I_{\sigma} \in \mathbf{I}_{l+1}$ and $\sigma(1) \leq a$ then $\Gamma_{D}\left(I_{\sigma}\right)=\Gamma_{E}^{*}\left(I_{\sigma}\right)$.

Note that condition 4 implies that $\gamma_{D}(\sigma)(1) \leq a$ for all $I_{\sigma} \in \mathbf{F}_{D}$ with $\sigma(1) \leq$ $a$.

Proof. The case $k=1$ is given by Lemma 6 .

Assume that $l=k+1$. Then $D^{\prime} \in M_{k}, D^{\prime} \subset U$, and $D^{\prime} \cap U_{1}=E$. Also, $D^{\prime \prime} \in M_{k-1}, \quad D^{\prime \prime} \subset U$, and $D^{\prime \prime} \cap U_{1}=E^{\prime}$. So Lemma 6 holds for $D^{\prime}$ and $D^{\prime \prime}$.

Let $p \in D \cap U_{x_{n}}$ for some $x_{n} \in A \cap X_{0}$. if $p \in \widehat{Q}_{k}$ then $f_{D}(p)=f_{D^{\prime}}(p) \in U_{x_{n}}$. Let $p \in Q_{1}$. Then $p$ is the midpoint of some element $I_{\sigma}$ of $\mathbf{I}_{k, n}$ where $\sigma(1)>c$. Also, $f_{D}(p)=h_{D^{\prime \prime}}(p)$ and $h_{D^{\prime \prime}}(p)$ is the midpoint of $\Gamma_{D^{\prime \prime}}\left(I_{\sigma}\right)$. But $\Gamma_{D^{\prime \prime}}\left(I_{\sigma}\right) \in \mathbf{J}_{k, n}$ and $\Gamma_{D^{\prime \prime}}(\sigma)(1)>b$. Thus $f_{D}(p) \in U_{x_{n}}$.

Let $p \in D \cap U_{q}$ for some $q \in A \backslash X_{0}$. Now $U_{q} \cap \widehat{Q}_{1} \subset\{q\}$ so $p=q$ and $p \in \widehat{Q}_{k}$. Thus $f_{D}(p)=f_{D^{\prime}}(p)=f_{E}(p) \in V$.

Let $I_{\sigma} \in \mathbf{F}_{D} \cap \mathbf{I}_{l+1, n}$ for some $x_{n} \in A \cap X_{0}$ and let $\sigma(1)>c$. Either $\gamma_{D}\left(I_{\sigma}\right)=\Gamma_{D^{\prime}}^{*}\left(I_{\sigma}\right)$ or $\gamma_{D}\left(I_{\sigma}\right)=\Gamma_{D^{\prime \prime}}^{*}\left(I_{\sigma}\right)$. In either case, $\gamma_{D}\left(I_{\sigma}\right) \in \mathbf{J}_{l+1, n}$ and $\gamma_{D}(\sigma)(1)>b>a$.

To show that conditions 3 and 4 hold, consider the sets $\mathbf{F}=\left\{\mathbf{I} \in I_{l+1}: I \subset\right.$ $\left.\bigcup \mathbf{F}_{E}\right\}$ and $\mathbf{G}=\left\{I_{\sigma} \in \mathbf{F}_{D}: \sigma(1) \leq a\right\}$. Define $\gamma$ on $\mathbf{G}$ by $\gamma(I)=\Gamma_{E}^{*}(I)$. We will show that $\mathbf{F} \subset \mathbf{G}$. Let $I_{\sigma} \in \mathbf{F}$. Then $\sigma(1) \leq a$ and $I_{\sigma\lceil k+1} \in \mathbf{F}_{E}$. Now $A(E) \subset A(D)$ because $E \subset D$. Also, $A(\theta(E)) \subset A(\theta(D))$. Thus $I_{\sigma} \in \widehat{\mathbf{F}}_{D}$ and $h_{D^{\prime}}\left(I_{\sigma}\right)=h_{E}\left(I_{\sigma}\right) \in \widehat{\mathbf{F}}_{\theta(D)}$. If $I_{\sigma} \in \mathbf{F}_{D 1}$ then there is $p \in D \cap Q_{l}$ such that $p$ is an endpoint of $I_{\sigma}$. Then, since $\sigma(1) \leq a, p \in U_{1}$. But $U_{1} \cap Q_{l}=\varnothing$, so $I_{\sigma} \notin \mathbf{F}_{D 1}$. If $p \in D \cap Q_{l}$ then $p \in U_{0}$ and $f_{D}(p) \in V_{0}$. But $\Gamma_{D^{\prime}}(\sigma)(1) \leq a$ so $h_{D^{\prime}}\left(I_{\sigma}\right)$ cannot have an endpoint in $\theta(D) \cap R_{l}$. Therefore $h_{D^{\prime}}\left(I_{\sigma}\right) \in \widehat{\mathbf{F}}_{\theta(D)} \backslash \mathbf{F}_{\theta(D) 1}$, and $I_{\sigma} \in \mathbf{G}$. By Lemma 3, $\Gamma(I)=\Gamma_{E}^{*}(I)$ for all $I \in \mathbf{I}_{l+1}$. If $I \in \mathbf{G}$ then $I \in \mathbf{F}_{D 2}$ so $\gamma_{D}(I)=\Gamma_{D^{\prime}}^{*}(I)=\Gamma_{E}^{*}(I)=\gamma(I)$. Thus $\gamma_{D}\left(I_{\sigma}\right) \in \mathbf{J}_{l+1, n}$ and $\gamma_{D}(\sigma)(1) \leq a<b$ for all $I_{\sigma} \in \mathbf{F}_{D} \cap \mathbf{I}_{l+1, n}$ with $m \leq \sigma(1) \leq b$. By Lemma 4, if $I_{\sigma} \in \mathbf{I}_{l+1, n}$ for some $x_{n} \in A \backslash X_{0}$ and $\sigma(1)>c$, then $\Gamma_{D}\left(I_{\sigma}\right) \in \mathbf{J}_{l+1, n}$ and $\Gamma_{D}(\sigma)(1)>b$. By Lemma 5, $\Gamma_{D}\left(I_{\sigma}\right)=\Gamma\left(I_{\sigma}\right)=\Gamma_{E}^{*}\left(I_{\sigma}\right)$ for all $I_{\sigma} \in \mathbf{I}_{l+1}$ with $\sigma(1) \leq a$.

Let $l \geq k+2$ and assume that if $j=l-1$ or $j=l-2, C \in M_{j}$, and $E \subset C \subset U$, then the lemma holds for $C$. Let $D \in M_{l}$ with $E \subset D \subset U$. Then $D \cap U_{1} \cap \widehat{Q}_{k+1}=E$. Furthermore $D^{\prime} \in M_{l-1}, E \subset D^{\prime} \subset U, D^{\prime \prime} \in M_{l-2}$, and $E \subset D^{\prime \prime} \subset U$. Thus the lemma holds for $D^{\prime}$ and $D^{\prime \prime}$. 
Let $p \in D \cap U_{x_{n}}$ for some $x_{n} \in A \cap X_{0}$. If $p \in \widehat{Q}_{l-1}$ then $f_{D}(p) \subset f_{D^{\prime}}(p) \in$ $V_{y_{n}}$. If $p \in Q_{l}$ then $p$ is the midpoint of some $I_{\sigma} \in \mathbf{I}_{l-1, n}$ with $\sigma(1)>c$. But $f_{D}(p)=h_{D^{\prime \prime}}(p)$ is the midpoint of $\Gamma_{D^{\prime \prime}}\left(I_{\sigma}\right)$ and $\Gamma_{D^{\prime \prime}}\left(I_{\sigma}\right) \in \mathbf{J}_{l-1, n}$ with $\Gamma_{D^{\prime \prime}}(\sigma)(1)>b$. Hence $f_{D}(p) \in V_{y_{n}}$.

Let $p \in D \cap U_{q}$ for some $q \in A \backslash X_{0}$. If $p \in \widehat{Q}_{l-1}$ then $f_{D}(p)=f_{D^{\prime}}(p) \in V$. If $p \in Q_{l}$ then $f_{D}(p)=h_{D^{\prime \prime}}(p)=\Gamma_{D^{\prime \prime}}^{*}(p)=\Gamma_{E}^{*}(p) \in V$ because $h_{E}\left(U_{q}\right) \subset V$.

Let $I_{\sigma} \in \mathbf{F}_{D} \cap \mathbf{I}_{l+1, n}$ for some $x_{n} \in A \cap X_{0}$ and let $\sigma(1)>c$. Either $\gamma_{D}\left(I_{\sigma}\right)=\Gamma_{D^{\prime}}^{*}\left(I_{\sigma}\right)$ or $\gamma_{D}\left(I_{\sigma}\right)=\Gamma_{D^{\prime \prime}}^{*}\left(I_{\sigma}\right)$. In either case, $\gamma_{D}\left(I_{\sigma}\right) \in \mathbf{J}_{l+1, n}$ and $\gamma_{D}(\sigma)(1)>b>a$.

To show that conditions 3 and 4 hold, consider the sets $\mathbf{F}=\left\{I \in \mathbf{I}_{l+1}: I \subset\right.$ $\left.\cup \mathbf{F}_{E}\right\}$ and $\mathbf{G}=\left\{I \in \mathbf{F}_{D}: \sigma(1) \leq a\right\}$. Define $\gamma$ on $\mathbf{G}$ by $\gamma(I)=\Gamma_{E}^{*}(I)$. Let $I_{\sigma} \in \mathbf{F}$. Then $I_{\sigma} \in \widehat{\mathbf{F}}_{D}$ because $E \subset D$ and $h_{D^{\prime}}\left(I_{\sigma}\right)=h_{E}\left(I_{\sigma}\right) \in \widehat{\mathbf{F}}_{\theta(D)}$ because $\theta(E) \subset \theta(D)$. Assume that $I_{\sigma} \notin \mathbf{F}_{D 1}$. Let $p \subset D \cap Q_{1}$. We will show that $f_{D}(p)$ cannot be an endpoint of $h_{D^{\prime}}\left(I_{\sigma}\right)$. If $p \in U_{0}$, then $f_{D}(p) \in V_{0}$. But $\Gamma_{D^{\prime}}(\sigma)(1) \leq a$ so $f_{D}(p)$ is not an endpoint of $h_{D^{\prime}}\left(I_{\sigma}\right)$. If $p \in U_{1}$ then $p \in I_{\tau}$ for some $I_{\tau} \in \mathbf{I}_{k+2}$ with $\tau(1) \leq a$. By the induction hypotheses, $f_{D}(p)=h_{D^{\prime \prime}}(p)=h_{E}(p) \in h_{E}\left(I_{\tau}\right)$. If $\sigma \mid k+2 \neq \tau$ then $I_{\sigma \nmid k+2} \cap I_{\tau}=\varnothing$ so $p$ cannot be an endpoint of any subinterval of $I_{\sigma \mid k+2}$. If $\sigma \mid k+2=\tau$ then $p$ is not an endpoint of $I_{\sigma}$ because $I_{\sigma} \notin \mathbf{F}_{D 1}$. The assumption that $I_{\sigma} \notin \mathbf{F}_{D 1}$ also implies that $h_{D}\left(I_{\sigma}\right)=h_{D^{\prime}}\left(I_{\sigma}\right)=h_{E}\left(I_{\sigma}\right)$. But $h_{E}^{-1}$ is continuous at $h_{E}(p)$, so $h_{E}(p)$ cannot be an endpoint of $h_{E}\left(I_{\sigma}\right)$. Therefore $h_{D}\left(I_{\sigma}\right) \in \widehat{\mathbf{F}}_{\theta(D)} \backslash \mathbf{F}_{\theta(D) 1}$ and $I_{\sigma} \in \mathbf{F}_{D 2}$. By Lemma 3, $\gamma(I)=\Gamma_{E}^{*}(I)$ for all $I \in \mathbf{I}_{l+1}$. If $I \in \mathbf{G}$ then either $\gamma_{D}(I)=\Gamma_{D^{\prime}}^{*}(I)$ or $\gamma_{D}(I)=\Gamma_{D^{\prime \prime}}^{*}(I)$. In either case, $\gamma_{D}(I)=\Gamma_{E}^{*}(I)=\gamma(I)$. Thus $\gamma_{D}\left(I_{\sigma}\right) \in \mathbf{J}_{l+1, n}$ and $\gamma_{D}(\sigma)(1) \leq a<b$ for all $I_{\sigma} \in \mathbf{F}_{D} \cap \mathbf{I}_{l+1, n}$ with $m \leq \sigma(1) \leq b$. By Lemma 4, if $I_{\sigma} \in \mathbf{I}_{l+1, n}$ for some $x_{n} \in A \cap X_{0}$ and $\sigma(1)>c$, then $\Gamma_{D}\left(I_{\sigma}\right) \in \mathbf{J}_{l+1, n}$ and $\Gamma_{D}(\sigma)(1)>b$. By Lemma 5, if $I_{\sigma} \in \mathbf{I}_{l+1}$ and $\sigma(1) \leq a$, then $\Gamma_{D}\left(I_{\sigma}\right)=\Gamma_{E}^{*}\left(I_{\sigma}\right)$.

Now let $B \in[A, U]$ and let $B \in S_{D}$. Then $D \in M_{l}$ for some $l \geq k$ and $E \subset D \subset U$. Also, $B \cap X_{0}=A \cap X_{0}$ so $\lambda\left(B \cap X_{0}\right)=\lambda\left(A \cap X_{0}\right) \subset V$. Let $p \in B \backslash X_{0}$. If $p \in D$ then $f_{D}(p) \in V$ by Lemma 7. Assume that $p \notin D$. There is $I_{\sigma} \in \mathbf{I}_{l+1}$ such that $p \in I_{\sigma}$. If $p \in U_{x_{n}}$ for some $x_{n} \in A \cap X_{0}$ then $I_{\sigma} \in \mathbf{I}_{l+1, n}$ and $\sigma(1)>c$. By Lemma $7, h_{D}\left(I_{\sigma}\right)=\Gamma_{D}^{*}\left(I_{\sigma}\right) \in \mathbf{J}_{l+1, n}$ and $\Gamma_{D}^{*}(\sigma)(1)>b$. Thus $h_{D}(p) \in V$. If $p \in U_{q}$ for some $q \in A \backslash X_{0}$ then $\sigma(1) \leq a$. By Lemma 7 , $h_{D}\left(I_{\sigma}\right)=\Gamma_{D}^{*}\left(I_{\sigma}\right)=\Gamma_{E}^{*}\left(I_{\sigma}\right)$. Thus $h_{D}(p) \in V$ because $h_{E}\left(U_{q}\right) \subset V$. Therefore $H(B) \in[H(A), V]$ and $H$ is continuous. A similar argument shows that $H^{-1}$ is continuous.

\section{RELATED RESUlts}

Corollary 8. If $X$ and $Y$ are $\omega$-graphs and $D$ and $E$ are equipotent discrete subsets of $X$ and $Y$ respectively, then $\bigcup_{p \in D}[p, X]$ is homeomorphic to $\bigcup_{p \in E}[p, Y]$. 
Proof. Extend $D$ and $E$ to dividing sets $X_{0}$ and $Y_{0}$ of $X$ and $Y$. Order the sets $X_{0}$ and $Y_{0}$ so that $\lambda(D)=E$. Then the homeomorphism defined in the proof of Theorem 1 takes $\bigcup_{p \in D}[p, X]$ to $\bigcup_{p \in E}[p, Y]$, so these two sets are homeomorphic.

The finally results are about spaces other than graphs or $\omega$-graphs. Theorem 2 of [N] shows that points may be removed from certain $T_{1}$ spaces without affecting its Pixley-Roy hyperspace. The next three lemmas generalize this result. Theorem 12 applies this procedure to $\mathbf{R}^{n}$.

Lemma 9. If $\left\langle Z_{n}: n\langle\omega\rangle\right.$ is a sequence of disjoint homeomorphic open and closed subsets of $\mathrm{PR}[X]$ such that $\bigcup_{n<\omega} Z_{n}$ is open and closed in $\mathrm{PR}[X]$, then $\operatorname{PR}[X] \backslash Z_{0} \approx \operatorname{PR}[X]$.

Proof. For each $n<\omega$ let $H_{n}: Z_{n} \rightarrow Z_{n+1}$ be a homeomorphism. Define $H: \operatorname{PR}[X] \rightarrow \operatorname{PR}[X] \backslash Z_{0}$ by

$$
H(A)= \begin{cases}A & \text { if } A \notin \bigcup_{n<\omega} Z_{n}, \\ H_{n}(A) & \text { if } A \in Z_{n} .\end{cases}
$$

Then $H$ is a homeomorphism.

Lemma 10. If $U$ is an open subset of space $X$ and $C$ is closed in $U$ then $\bigcup_{p \in C}[p, U]$ is open and closed in $\operatorname{PR}[X]$.

Proof. Clearly $\bigcup_{p \in C}[p, U]$ is an open subset of $\operatorname{PR}[X]$. Let

$$
A \in U \backslash \bigcup_{p \in C}[p, U] \text {. }
$$

If $A \not \subset U$ then $[A, X]$ is a neighborhood of $A$ that misses $\bigcup_{p \in C}[p, U]$. If $A \subset U$ then $A \cap C=\varnothing$, so $[A, U \backslash C]$ is a neighborhood of $A$ in $\operatorname{PR}[X]$ that misses $\bigcup_{p \in C}[p, U]$.

Lemma 11. Let $\left\langle U_{n}: n<\omega\right\rangle$ be a sequence of disjoint open subsets of a space $X$ and let $\left\langle C_{n}: n<\omega\right\rangle$ be a sequence of subsets of $X$ such that $C_{n} \subset U_{n}$ and $C_{n}$ is closed in $U_{n}$ for all $n<\omega$. Then $\bigcup_{n<\omega} \bigcup_{p \in C_{n}}\left[p, U_{n}\right]$ is open and closed in $\mathrm{PR}[X]$.

Proof. It is clear that $\bigcup_{n<\omega} \bigcup_{p \in C_{n}}\left[p, U_{n}\right]$ is open in PR $[X]$. By Lemma 10, each $\bigcup_{p \in C_{n}}\left[p, U_{n}\right]$ is closed in PR $[X]$. Let $A \in \operatorname{PR}[X]$. Since $A$ is finite and the $U_{n}$ 's are disjoint, there is a finite subset $B$ of $\omega$ such that $A \cap U_{n} \neq \varnothing$ if and only if $n \in B$. Then $\left(\bigcup_{m \in B}\left[A, U_{m}\right]\right) \cap\left(\bigcup_{p \in U_{n}}\left[p, U_{n}\right]\right) \neq \varnothing$ only if $n \in B$. Thus $\left\{\bigcup_{p \in C_{n}}\left[p, U_{n}\right]: n<\omega\right\}$ is locally finite, and $\bigcup_{n<\omega} \bigcup_{p \in C_{n}}\left[p, U_{n}\right]$ is closed.

Theorem 12. Let $0<n<\omega$ and let $X=\left\{\bar{x} \in R^{n}: 0<|\bar{x}|<1\right\}$ where $|\bar{x}|$ denotes the Euclidean norm. For any $0<m<\omega$,

$$
\mathrm{PR}\left[\mathbf{R}^{n}\right] \approx \mathrm{PR}\left[m \times \mathbf{R}^{n}\right] \approx \operatorname{PR}\left[\omega \times \mathbf{R}^{n}\right] \approx \operatorname{PR}[m \times X] \approx \operatorname{PR}[\omega \times X] .
$$

Pronf. We will show that each of these spaces is homeomorphic to $\mathrm{PR}\left[\mathbf{R}^{n}\right]$. Let $D$ be a discrete subset of $\{x \in \mathbf{R}: x \geq 0\}$ which contains 0 and let $\pi: \mathbf{R}^{n} \rightarrow \mathbf{R}$ 
be the projection onto the first coordinate. Let $L=\left\{\bar{x} \in R^{n}: \pi(\bar{x}) \in D\right\}$ and let $C=\left\{\bar{x} \in \mathbf{R}^{n}:|\bar{x}| \in D\right\}$. If $D$ is finite then $\mathbf{R}^{n} \backslash L=(|D|+1) \times \mathbf{R}^{n}$ and $\mathbf{R}^{n} \backslash C=|D| \times X$. If $D$ is infinite then $\mathbf{R}^{n} \backslash L \approx \omega \times \mathbf{R}^{n}$ and $\mathbf{R}^{n} \backslash C \approx \omega \times X$. Let $U_{0}=\mathbf{R}^{n}$ and let $\left\langle U_{k}: 0<k<\omega\right\rangle$ be a sequence of disjoint open balls in $\mathbf{R}^{n}$, each of which has empty intersection with $L$ and $C$.

Set $C_{0}=L$. For every $0<k<\omega$ let $C_{k}$ be a subset of $U_{k}$ which is homeomorphic to $L$. Then $C_{k}$ is closed in $U_{k}$ for all $k<\omega$. For each $k<\omega$ set $Z_{k}=\bigcup_{p \in C_{k}}\left[p, U_{k}\right]$. By Lemma 10 , each $Z_{k}$ is open and closed in $\mathrm{PR}\left[\mathbf{R}^{n}\right]$. By Lemma $11, \bigcup_{0<k<\omega} Z_{k}$ is open and closed in $\operatorname{PR}\left[\mathbf{R}^{n}\right]$, so $\bigcup_{k<\omega} Z_{k}$ is open and closed in $\operatorname{PR}\left[\mathbf{R}^{n}\right]$. Clearly each $Z_{k}$ is homeomorphic to every other $Z_{k}$, so $\operatorname{PR}\left[\mathbf{R}^{n}\right] \approx \operatorname{PR}\left[\mathbf{R}^{n}\right] \backslash Z_{0} \approx \operatorname{PR}\left[\mathbf{R}^{n} \backslash L\right]$. If $D$ is finite then $\operatorname{PR}\left[\mathbf{R}^{n}\right] \approx \operatorname{PR}\left[(|D|+1) \times \mathbf{R}^{n}\right]$. If $D$ is infinite then $\operatorname{PR}\left[\mathbf{R}^{n}\right] \approx \operatorname{PR}\left[\omega \times \mathbf{R}^{n}\right]$.

Now let $C_{0}=C$ and for every $k<\omega$ let $C_{k}$ be a subset of $U_{k}$ homeomorphic to $C$. Set $Z_{k}=\bigcup_{p \in C_{k}}\left[p, U_{k}\right]$ for all $k<\omega$. Again, $\left\langle Z_{k}: k<\omega\right\rangle$ is a sequence of disjoint homeomorphic open and closed subsets of $\operatorname{PR}\left[\mathbf{R}^{n}\right]$ so $\operatorname{PR}\left[\mathbf{R}^{n}\right] \approx \operatorname{PR}\left[\mathbf{R}^{n}\right] \backslash Z_{0} \approx \operatorname{PR}\left[\mathbf{R}^{n} \backslash C\right]$. If $D$ is finite then $\operatorname{PR}\left[\mathbf{R}^{n}\right] \approx \operatorname{PR}[|D| \times X]$. If $D$ is infinite then $\operatorname{PR}\left[\mathbf{R}^{n}\right] \approx \mathrm{PR}[\omega \times X]$.

\section{BIBLIOGRAPHY}

[D] Peg Daniels, Pixley-Roy spaces over subsets of the reals, Topology Appl. 29 (1988), 93-106.

[vD] E. K. van Douwen, The Pixley-Roy topology on spaces of subsets, Set-Theoretic Topology, Academic Press, New York, 1977, pp. 111-134.

[L] D. J. Lutzer, Pixley-Roy topology, Topology Proc. 3 (1978), 139-158.

[N] Jeffrey Norden, Pixley-Roy spaces over graphs, Topology Appl. (to appear).

[W] M. L. Wage, Homogeneity of Pixley-Roy spaces, Topology Appl. 28 (1987), 45-57.

Department of Mathematics, University of Dayton, Dayton, Ohio 45469 